\title{
Is Always a Hybrid Recommender System Preferable To Single Techniques?
}

\author{
Himan Abdollahpouri \\ Iran University of Science and \\ Technology \\ Tehran, \\ Iran
}

\author{
Adel Rahmani \\ Iran University of Science and \\ Technology \\ Tehran, \\ Iran
}

\author{
Alireza Abdollahpouri \\ University of Kurdistan \\ Sanandaj, \\ Iran
}

\begin{abstract}
Collaborative filtering (CF) recommender systems are typically unable to generate adequate recommendations in sparse datasets. Empirical evidence suggests that incorporation of a trust network among the users of a recommender system can significantly help to alleviate this problem. For this reason, some studies have been done on combining $\mathrm{CF}$ with trust-enhanced recommender system. In this study, we analyze the switching hybrid recommender system with the $\mathrm{CF}$ and trust-enhanced recommender system components from both rating coverage and mean absolute error point of view. Experiments on a dataset from Epinions.com prove that, although the rating coverage of this hybrid method is better than both (CF and trust-enhanced RS), but has lower accuracy than just using trust-enhanced RS. In other words, trust-enhanced RS outperforms the hybrid recommender system consisting of CF and trust-enhanced RS. Finally, we justify this result using analytical method.
\end{abstract}

\section{General Terms}

Information filtering,; machine learning; information retrieval

\section{Keywords}

Recommender systems; collaborative filtering; trust; hybrid recommender system

\section{INTRODUCTION}

Recommender systems can guide the users through the vast amount of information, and they are gaining tremendous importance in recent years. These systems receive some information about their user's profiles and relationships, and then, suggest items that might be of interest to them [6][25]. A variety of techniques have been proposed for performing recommendation, including content-based, collaborative, knowledge-based and other techniques. To improve performance, these methods have sometimes been combined in hybrid recommenders. One common thread in recommender systems research is the need to combine recommendation techniques to achieve peak performance. All of the known recommendation techniques have strengths and weaknesses, and many researchers have chosen to combine techniques in different ways. Hybrid recommender systems combine two or more recommendation techniques to gain better performance with fewer of the drawbacks of any individual one. Most commonly, collaborative filtering is combined with some other technique in an attempt to avoid the ramp-up problem [20]. One of the most widely used recommendation techniques is collaborative filtering (CF), which typically works by identifying users whose tastes are similar to those of a particular user and by recommending items that they have liked [3][7][26]. However, CF recommender systems face important challenges. One of the most important is data sparsity [9]. Data sparsity indicates the cases when the number of ratings available, are significantly low in comparison with the number of items and users. In this situation, finding similar users is impossible for some users; because they have not rated sufficient items and similarity equations such as Pearson Correlation Coefficient (PCC) cannot be applied [6][25]. One of the promising directions suggests that the incorporation of a trust network (in which users are connected by trust scores indicating how much they trust and/or distrust each other), can significantly help to alleviate this problem. Primarily, it is because the information calculated in trust statements about RS's users can be propagated and aggregated, and hence more people and products can be matched [8][16][17][18].

There are several studies on combining collaborative filtering with other recommender system techniques and its advantage of alleviating the CF problems [17][18][20]. In this paper, we prove that, combining two different recommender systems will not improve the performance of both techniques necessarily. We analyze the combination of $\mathrm{CF}$ and trustbased recommender system in both rating coverage and Mean Absolute Error (MAE) point of view (these evaluation metrics will be discussed in Section 4) on a real dataset called Epinions. Epinions is a real dataset and has the characteristics of real data such as high sparsity.

We know that in real world of recommender systems, the dimension of data is really high with millions of rows and columns, so it is important to do all the analysis and calculations on a dataset which is similar to real data. We prove that, trust-enhanced RS outperforms combination of $\mathrm{CF}$ and trust-enhanced recommender system from the accuracy point of view. It means that, the MAE of trust-enhanced RS is lower than the MAE of this hybrid method, hence has higher accuracy. Finally, we justify this result using analytical method.

The rest of the paper is organized as follows: In Section 2, we present some related work on recommender systems. In Section 3, we describe the combination of $\mathrm{CF}$ and trustenhanced recommender system. Section 4 describes our dataset used in this study and its characteristic and after that we explain some evaluation metrics used to qualification of recommender systems and at the end of this section, we give our experimental results and then, prove them with an analytical approach. Finally, we conclude the paper in Section 5 .

\section{RELATED WORK}

Recommender systems are often used to accurately estimate the degree to which a particular user will like a specific item. Such algorithms come in many forms, such as content-based, 
collaborative filtering and trust-based methods; the latter two methods are most relevant to our current effort [19][25].

\subsection{Classical CF Recommender System}

$\mathrm{CF}$ algorithms produce a rating for an item $\mathrm{i}$ which is new to a user a. This new rating is based on a combination of the ratings of the nearest neighbours (similar users) already familiar with item i [2]. The classical CF-formula is given by (1). The unknown rating $\mathrm{Pa}, \mathrm{i}$ for an item $\mathrm{i}$ for a user a is predicted based on the mean of ratings by a for other items, as well as on the ratings ru,i by other users $u$ for $i$. The formula also takes into account the similarity wa, $\mathrm{u}$ between users a and ${ }^{a} \mathrm{u}$, usually calculated as Pearson's Correlation Coefficient (PCC):

$$
P_{a, i}=\bar{r}_{a}+\frac{\sum_{u \in U}\left(r_{u, i}-\bar{r}_{u}\right) \cdot w_{a, u}}{\sum_{u \in U}\left|w_{a, u}\right|}
$$

Throughout this paper, the rating coverage for a user a, or coverage for short, refers to the ratio of the amount of items for which $\mathrm{Pa}, \mathrm{u}$ as in (1) can be calculated versus the total amount of items available in the RS. For any RS algorithm, an increment in coverage is only beneficial when the accuracy does not drop significantly, while an accuracy increase is not useful when there are too few ratings that can be predicted. Hence, coverage and accuracy should be evaluated together.

The effectiveness (accuracy and coverage) of CF-based RSs is significantly affected by the number of ratings available for each user. The more ratings are available, the better the quality of the recommendations. Moreover, generating recommendations is only possible for users who have rated at least two items; because the PCC requires at least two ratings per user [2][7][16]. An important problem in CF is data sparsity (DS problem). In practice, many commercial recommender systems are used to evaluate very large product sets. The user-item matrix used for collaborative filtering will thus be extremely sparse and the performances of the predictions or recommendations of the $\mathrm{CF}$ systems are challenged. Most of the approaches combine rating data with content data to alleviate DS problem, such as Middleton et al. [23] who work with information delivered by ontologies, and Park et al. [24] who focus on simple filterbots. Ahn [7] and Huang et al. [26], only use rating data: the former introduces a similarity measure which takes into account the proximity of ratings, the rating impact and item popularity, while in the latter approach the set of $\mathrm{CF}$ neighbours is extended by exploring transitive associations between the items and users.

\subsection{Trust-enhanced RS}

Trust-enhanced RSs can alleviate the DS problem by using additional information coming from a trust network in which the users are connected by trust scores indicating how much they trust and/or distrust each other [9][15]. Such trust networks can be generated automatically, e.g. inferred through the similarity of rating behaviour or based on a user's history of making reliable recommendations. Another approach is to ask RS's users explicitly to issue trust statements about users. A well-known trust-enhanced example is Epinions.com, an ecommerce site where users can rate products and include users in their personal web of trust. In [18], Messa et al. investigate how trust can be incorporated into the CF process by conducting experiments on a dataset from Epinions. They propose a special case of (1) in which the weights wa,u are replaced by trust information ta, $u$. The formula is given in (2). In this approach, trust is interpreted as a numerical value which ranges between 0 and 1 , denoting absence and full presence of trust, respectively[11][13].

$$
P_{u, i}=\overline{r_{u}}+\frac{\sum_{a \in T(u)} t_{a, u}\left(r_{a, i}-\overline{r_{a}}\right)}{\sum_{a \in T(u)} t_{a, u}}
$$

\subsubsection{Trust inferences}

Due to the number of ratings that exist in recommendation systems, underlying social networks are very sparse. There are cases in which insufficient or loss of information is detrimental for the recommendation algorithms. Consider, for example, the case in which associations between users are based on very few data or the case in which there aren't any $k$ users to employ in a k-nearest neighborhood algorithm. A motivating example is illustrated in Fig. 1. Suppose that users $\mathrm{S}, \mathrm{N}$ have rated item $\mathrm{I} 1$ and users $\mathrm{N}, \mathrm{T}$ have rated $\mathrm{I} 2$. Classic $\mathrm{CF}$ will associate user $\mathrm{S}$ with user $\mathrm{N}$ and user $\mathrm{N}$ with user $\mathrm{T}$, but not user $\mathrm{S}$ with user T. However, a more sophisticated approach that incorporates transitive interactions would recognize the associative relationship between user $\mathrm{S}$ and user $\mathrm{T}$ and infer this indirect association. To deal with this problem, we can adopt a method of inferring trust between users that are not directly associated to each other.Thus, in the example, it is possible to infer trust between the source user $\mathrm{S}$ and the target user $\mathrm{T}$ through the intermediate user $\mathrm{N}$. According to this process, trust is propagated in the network and associations between users are built, even if they have no co-rated item. The main strength of trust-enhanced recommender systems is their use of trust propagation operators; mechanisms to estimate the trust transitively by computing how much trust a user a has to another user c, given the value of trust for a trusted third party (TTP) b by a, and $c$ by $b$. Although there is much debate about the most suitable propagation operator(s), see e.g. [8][21][22], all of them agree on the case of atomic direct propagation, namely that if a trusts $b$ and $b$ trusts $c$ then it is inferred that a trusts $c$.

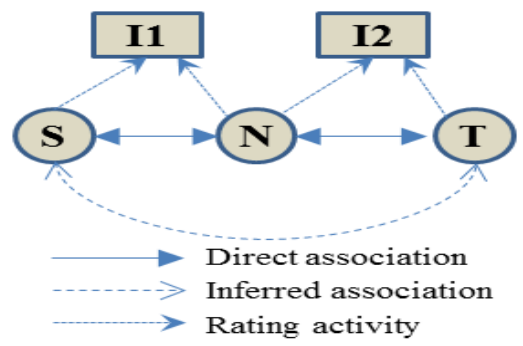

Fig. 1 Trust inferences [14]

Goldbeck's Tidaltrust and Messa's MoleTrust are specifically designed for propagation of trust only [10][18]. They both choose multiplication as propagation operator and take into account a maximum propagation depth and a minimum trust value below which users are not allowed to interfere in the recommendation process, but the ways these two thresholds are determined differ significantly. Another, very recent research path is the propagation of trust and distrust, which obviously requires new propagation operators. By combining (propagated) trust information with the available ratings, more users and (consequently) more items get covered by the RS, even if only few trust statements per user are available. In 
particular, a prediction $\mathrm{Pa}, \mathrm{u}$ can be calculated when a trust at least one user $\mathrm{u}$ to a degree $t_{a, u} \neq 0$ and $\mathrm{u}$ already rated $\mathrm{i}$.

\section{COMBINING COLLABORATIVE FILTERING AND TRUST-ENHACED RS}

There are several approaches to make a hybrid recommender system. One of them is weighted hybrid recommender system that combines different recommendation techniques by their weights, which are computed from the results of all of the available recommendation techniques present in the system. The combination can be linear, the weights can be adjustable, and weighted majority voting or weighted average voting can be used. Other methods for making a hybrid recommender system such as switching, mixed, feature combination, cascade, feature augmentation and meta-level can be found in [20]. The method which we have used in this paper is switching hybrid recommender system. This approach switches between recommendation techniques using some criteria, such as confidence or any other criteria levels for the recommendation techniques. In our case, when the $\mathrm{CF}$ system cannot make a recommendation according to these criteria, then, another recommender system such as trust-enhanced RS is attempted. We have considered the ability to calculate similarity as our switching criterion. The approach of combining CF with trust-enhanced RS is as follows: first we use $\mathrm{CF}$ to find similar users to a specific user. If similar users are found, we use their ratings as an estimation of predicted rating for the items that the user has not rated. But if similarity cannot be calculated, we use trust information and find users who trusted by this user. The architecture of combining collaborative filtering with Trust-enhanced recommender system is depicted in Fig. 2. The "rating prediction" module takes as input both the estimated trust matrix and the user similarity matrix. The idea is that the weight of a neighbour used in Eq. (1) can be derived both from the user similarity value computed by the similarity metric (Pearson Correlation Coefficient in our case) or the predicted trust value computed by a trust metric. If the former cannot be calculated, the latter will be used. First, we compare performances of RS algorithms that use only trust information with standard CF. We start by using only users explicitly trusted by the active user (the user whom we want to recommend for), i.e., not propagating trust or setting the propagation horizon at 1 for the local Trust metric MoleTrust [17]. This algorithm is called MT1. In general RSs based on trust propagation, work better in sparse datasets. They don't use (few) ratings information for deriving a similarity measure to be used as weight for that user, but use the trust information explicitly provided by the user. In this way, even for a user with just one friend, it is possible that her/his friend has rated the items she/he rated and hence a prediction is possible.

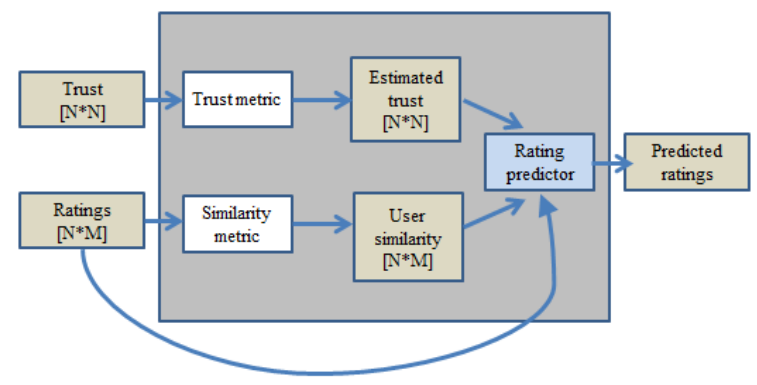

Fig. 2. Hybrid recommender system architecture consists of CF and trust-enhanced RS

\section{EMPIRICAL VALIDATION AND EXPERIMENTS RESULTS}

In this section, we present experiments conducted by us for evaluating the performance of the switching hybrid recommender system with $\mathrm{CF}$ and trust-enhanced $\mathrm{RS}$ components. In particular, we compare combination of $\mathrm{CF}$ and trust-enhanced RS with systems using only trust metrics and systems that use pure collaborative filtering. First, we describe the dataset and introduce the evaluation strategy we followed. Then, we present the actual results of the experiments.

\subsection{Epinions.com dataset}

The dataset we used in our experiments is derived from the Epinions.com web site. Epinions is a consumers opinion site where users can review items (e.g., cars, books, movies, softwares) and also assign them numeric ratings in the range of $1(\min )$ to $5(\max )$. Users can also express their web of trust, i.e. reviewers whose reviews and ratings they have consistently found to be valuable and their block list, which is a list of authors whose reviews they find consistently offensive, inaccurate, or not valuable. Inserting a user in the web of trust equals to issuing a trust statement of value 1 (max trust) in her/him while inserting her in the block list equals to issuing a trust statement of value 0 (min trust) in her/him. Intermediate values such as 0.6 are not expressible in Epinions. Note however that the block list is kept private in Epinions.com in order to let users express more freely, so it is not available in this dataset. The dataset consists of 49,290 users who rated a total number of 139,738 different items at least once. The total number of reviews is 664,824 and the total number of issued trust statements is 487,181 . Rating matrix sparsity is defined as the percentage of empty cells in the matrix user-items and in this case it is $99.99135 \%$. The mean number of created reviews is 13.49 with a standard deviation of 34.16. It is interesting to have a look at what we called "cold start users". They are the large majority of users. For example, 26,037 users expressed less than 5 reviews and they account for $52.82 \%$ of the population which is shown in Fig. 3. The mean number of users in the web of trust is 9.88 with a standard deviation of 32.85 . Fig. 4 shows the number of users who expressed different number of trust statements; another interesting point is the distribution of ratings. About $45 \%$ of the ratings are 5 , and $29 \%$ are 4 . For the case of 3 ratings, this value is $11 \%$. This percentage is 8 and 7 for 2 and 1 rating(s), respectively. The mean rating is hence 3.99 . Note that almost half of the ratings are 5, i.e., the maximum possible value.

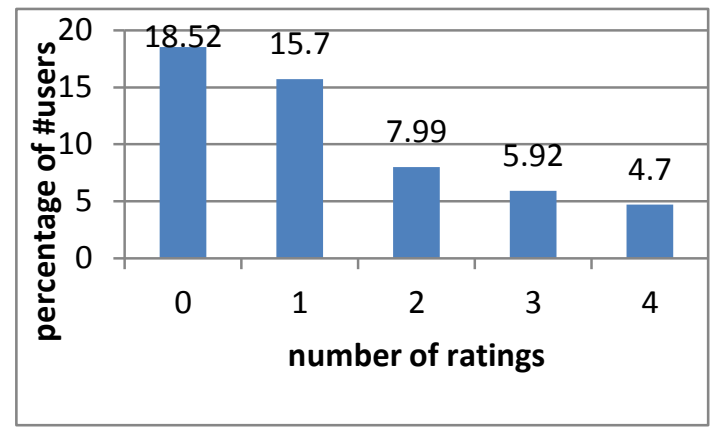

Fig. 3. The percentage of users who have rated $0,1,2,3,4$ items. 


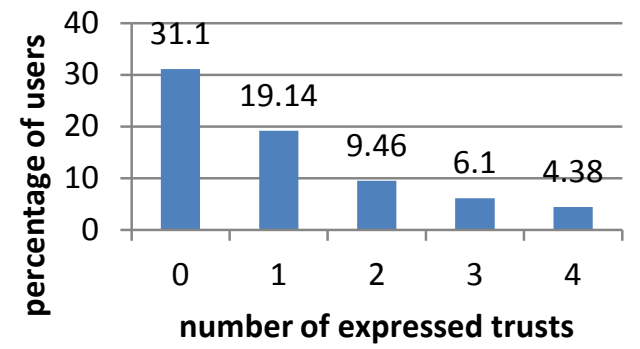

Fig. 4. The percentage of users who have expressed $0,1,2,3,4$ trust statements. The percentage of users who have expressed less than 4 trust statements is $70.18 \%$

\subsection{Evaluation Metrics}

The most widely used technique for evaluating recommender systems is based on leave-one-out [3][11][12]. Leave-one-out is an offline technique that can be run on a previously acquired dataset and involves hiding one rating and then trying to predict it with a certain algorithm. The predicted rating is then compared with the real rating and the absolute value of the difference is the prediction error. The procedure is repeated for all the ratings and an average of all the errors is computed as the Mean Absolute Error (MAE). Another important measure that is often not reported and studied in evaluation of RSs is coverage. Herlcker et al., in their solid review of recommender systems evaluation techniques, underline how it is important to go "beyond accuracy" in evaluating RSs [12]. They count coverage as one step in this direction. Coverage simply refers to the fraction of ratings for which, after being hidden, the RS algorithm is able to produce a predicted rating. It might in fact be the case that some RS techniques are not able to predict the rating a user would give to an item. These two evaluation metrics belong to the objective metrics for measuring the quality of a recommender system. There are also subjective metrics that we can take into account and enhance the user experience according to these subjective metrics [4]. Diversity, explanation, transparency, robustness are some of most important subjective metrics that should be measured carefully in order to evaluate the RS techniques [13]. In this paper, we only focus on objective metrics, i.e. MAE and coverage.

\subsection{Experiments Results}

$\mathrm{CF}$ uses the ratings of items rated by users to calculate similarity between them. That means it has no idea about what exactly one user trust on another user. This problem would be more dramatic when the number of items a specific user has rated is few. Hence deciding on these few ratings for calculating similarity between users seems to be not very accurate. From this, we can conclude that, finding similarity between users or items just based on ratings would cause our RS to have weak similarity computation in some situations that the number of ratings is not sufficient. Since similarity computation is the vital step in recommendation process, hence recommender system would make weak recommendation; and as a result, would have low accuracy. In fact, in real datasets such as Epinions, we have this situation. As we mentioned in subsection 4.1, this dataset is $99.99 \%$ sparse. Implementing the combination of $\mathrm{CF}$ with trustenhanced RS, we achieve the results depicted in Fig. 5 and Fig. 6. From Fig. 5, we can see that the MAE of CF is higher than other two methods (trust-enhanced method and the hybrid method). The interesting point that we should notice is that the accuracy of hybrid method is higher than typical CF and lower than trust-enhanced RS. On the other hand, from Fig. 6, we can see that the rating coverage of the combination method is higher than two other methods, since in this case we have both ratings that $\mathrm{CF}$ can predict and those that $\mathrm{CF}$ cannot, but trust-enhanced RS can. Improvement of rating coverage was predictable but what happened to MAE? Why the MAE of hybrid method is higher than trust-enhanced RS? To justify these results we are going to find a formula to calculate the MAE of this hybrid method. First, we denote the ratings that $\mathrm{CF}$ can predict with $\mathrm{PCF}$ and the ratings that trustenhanced RS can predict with PTrust. Being more formally:

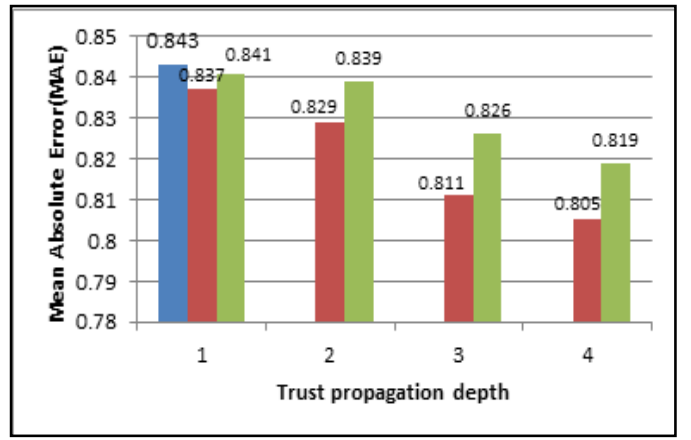

a $\quad$ trust-enhanced $\quad$ CF with trust-enhanced

Fig. 5. The MAE of different RS algorithms: CF,Trustenhanecd, combination of CF and Trust-enhanced RS with switching approach

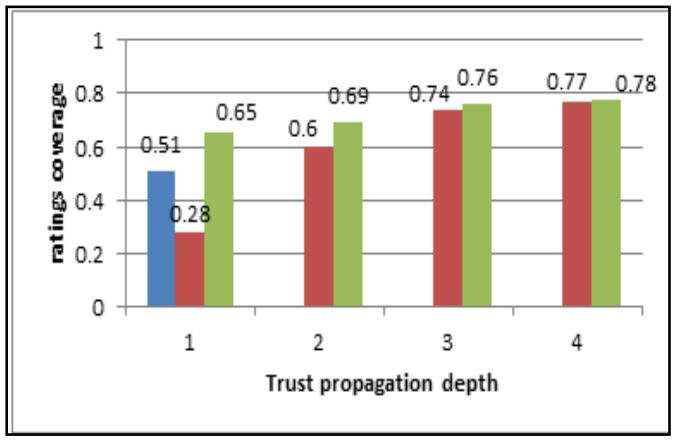

Fig. 6. The rating coverage of different $R S$ algorithms: $C F$, Trustenhanced, combination of CF and Trust -enhanced RS with switching approach.

$$
\begin{aligned}
& P_{C F}=\left\{p_{1}, p_{2}, p_{3}, \ldots, p_{L-1}, p_{L}\right\} \\
& P_{\text {Trust }}=\left\{t_{1}, t_{2}, t_{3}, \ldots, t_{Z-1}, t_{Z}\right\}
\end{aligned}
$$

This notification tells us that the ratings that CF can predict, is a set of $L$ ratings and the ratings that trust-enhanced $R S$ can predict is a set of $\mathrm{Z}$ ratings. $\mathrm{L}$ and $\mathrm{Z}$ are positive integer values. Hence, the ratings that combination method can predict will be Pcombination::

$$
P_{\text {combination }}=P_{C F}+G ; G \subset P_{\text {Trust }}
$$

$\mathrm{G}$ is the subset of PTrust because the ratings that cannot be predicted by $\mathrm{CF}$, is calculated by trust-enhanced RS and if 
calculable, it will be the predicted rating by hybrid method. If we denote the number of whole ratings as $\mathrm{A}$ then the number of ratings that $\mathrm{CF}$ cannot predict but trust-enhanced $\mathrm{RS}$ can, will be (A-L)m . Here, $\mathrm{L}$ is the number of ratings that $\mathrm{CF}$ can predict and $\mathrm{m}$ is the variable coefficient that is between 0 and 1. The MAE of CF and Trust-enhanced RS can be calculated using the following equations:

$$
M A E_{C F}=\frac{\sum_{i=1}^{L} p_{i}}{L} \quad M A E_{\text {trust }}=\frac{\sum_{i=1}^{Z} t_{i}}{Z}
$$

according to this dataset. In our dataset (Epinions.com) the following fact holds:

$$
L \cong \frac{A}{2} \Rightarrow A \cong 2 L
$$

To explain this fact, it is enough to look at Fig. 5; according to this figure, the $\mathrm{CF}$ rating coverage is $51 \%$ that can be approximated as $50 \%$, so $\mathrm{L}$ is half of $\mathrm{A}$ ( the number of whole ratings available in the dataset). This means that, $\mathrm{CF}$ can predict only half of ratings available in the dataset, so the Eq. (7) takes the following form:

Table.1 Number of ratings that different algorithms can predict on Epinions dataset

\begin{tabular}{cccccccc}
\hline CF & Trust1 & Trust2 & Trust3 & Trust4 & $\begin{array}{c}\text { Number of } \\
\text { ratings }\end{array}$ & $\begin{array}{c}\text { MAE of using } \\
\text { trust algorithm }\end{array}$ & $\begin{array}{c}\text { Corresponding } \\
\text { value of } m\end{array}$ \\
\hline 1 & DC & DC & DC & DC & $\mathbf{3 3 9 0 6 0}$ & DC & DC \\
DC & 1 & DC & DC & DC & 186150 & 0.837 & DC \\
DC & DC & 1 & DC & DC & 398894 & 0.829 & DC \\
DC & DC & DC & 1 & DC & 491969 & 0.811 & DC \\
DC & DC & DC & DC & 1 & 511914 & 0.805 & DC \\
0 & 1 & DC & DC & DC & $\mathbf{9 3 0 7 5}$ & 0.833 & $\mathbf{0 . 2 8 5 7}$ \\
0 & DC & 1 & DC & DC & 119668 & 0.827 & 0.3673 \\
0 & DC & DC & 1 & DC & 166206 & 0.791 & 0.5102 \\
0 & DC & DC & DC & 1 & 179502 & 0.774 & 0.5510 \\
\hline
\end{tabular}

For calculating MAE of hybrid method we should take into account the MAE of CF and Trust-enhanced RS. Also we should know that the number of ratings that hybrid method can predict may higher than both CF and trust-enhanced RS. We know that MAE is the average of predicted ratings errors. In this case it will be the average of Pcombination errors. Hence the MAE of Pcombination will be:

$M A E_{\text {combination }}=\frac{\sum_{i=1}^{L} p_{i}}{L+m(A-L)}+\frac{\sum_{j=x}^{x+m(A-L)-1} t_{j}}{L+m(A-L)}$

where, $x$ is an integer value and has a value between 1 and $\mathrm{Z}$ ( it is an index in $P_{\text {Trust }}$ set). From Eq. (5) and Eq. (6) we have:

$$
M A E_{\text {combination }}=\frac{M A E_{C F}}{1+\frac{m(A-L)}{L}}+\frac{m(A-L) \times M A E_{G}}{L+m(A-L)}
$$

Eq. (7) is true for any dataset. It means that in any dataset when we combine CF with trust-enhanced RS with switching approach then the MAE of result will be in the form of Eq. (7). As we mentioned in Section 4, we used Epinions dataset for our experiment. It is clear that, for rejection of a claim, it is enough to give an example that shows the claim is not always true. So from now, we continue our justification

$$
\begin{aligned}
& M A E_{\text {combination }}=\frac{1}{m+1}\left(M A E_{C F}+m \times M A E_{G}\right) \\
& 0 \leq m \leq 1
\end{aligned}
$$

Remember again that the fact $\mathrm{A}=2 \mathrm{~L}$ holds in Epinions dataset and it is not necessarily true for other datasets. Eq. (9) demonstrates that, MAE of the hybrid method depends on the ratio of ratings that $\mathrm{CF}$ cannot predict but trust-enhanced RS can in the Epinions dataset. To find this ratio, we should come back to our dataset again and find the ratings. Table 1 gives some useful information about the number of ratings that $\mathrm{CF}$ and trust-enhanced RS can predict or not. In this table, you can see the number of ratings that $\mathrm{CF}$ can predict with the consideration of other algorithms (trust1, trust2, trust 3 and trust4) combined with it. In addition, the number of ratings that each algorithm can predict independently is also presented. The MAE of using trust algorithm with different trust propagation depths on these ratings is also shown. This information can be calculated for any dataset and the results can be injected into Eq. (9) and the MAE can be found. DC stands for don't care and it means that the corresponding algorithm is not considered. For example, the first row is the number of ratings that $\mathrm{CF}$ can predict without considering other algorithms (Trust1, Trust2, Trust3, and Trust4) and the last row shows the number of ratings that $\mathrm{CF}$ cannot predict but Trust 4 can, without considering other algorithms (Trust1,Trust2,Trust3), and the MAE of using Trust4 on this 
ratings. According to this information, the MAE of combining CF with Trust 1 can be calculated as follows:

$$
M A E_{\text {combination }}(\text { Trust } 1)=\frac{1}{m+1}(0.843+m \times 0.815)
$$

Since $\mathrm{m}$ is the ratio of ratings that $\mathrm{CF}$ cannot predict but Trust1 can (in this case we use Trust1), hence it will be $93075 /(664824-339060)=1 / 3.5$ i.e. $\mathrm{m}$ is 0.2857 . Remember that, number of whole ratings are 664824 and the number of ratings that $\mathrm{CF}$ can predict is 339060 . Also the number of ratings that $\mathrm{CF}$ cannot predict but trust 1 can is 93075 . So the MAEcombination

$$
\text { will }
$$

be $(0.843+0.2857 \times 0.815) / 1.2857=0.841$. The MAE of combining CF with different trust methods (Trust1, Trust2, Trust3, and Trust4) was shown previously in Fig. 5. The corresponding value of $\mathrm{m}$ for different trust method is shown in most right column of Table 1. This result was not far unpredictable, since $\mathrm{CF}$ is not able to find good neighbours, more participating in prediction process will cause to accuracy get lower and hence MAE will be higher. In the case of rating coverage, it is obvious that the coverage of combining $\mathrm{CF}$ with trustenhanced RS (or any other recommender system) is higher than or equal to $\mathrm{CF}$; when ratings that $\mathrm{CF}$ cannot predict also cannot be calculated by trust-enhanced RS, then rating coverage of $\mathrm{CF}$ and the hybrid method would be equal; in other cases, it will be higher for hybrid method. But according to Fig. 6, this improvement in rating coverage is not much higher than trust-enhanced RS rating coverage for trust propagation depths 2, 3, 4 and higher.

\section{CONCLUSION}

In this paper, we investigated the accuracy of the combination $\mathrm{CF}$ and trust-enhanced recommender system. We used switching approach for this combination. That means when one criterion did not meet (in our case this criterion is calculating similarity) we switch to another method. In fact, we first try $\mathrm{CF}$, and whenever $\mathrm{CF}$ cannot find similar users to active user, try trust-enhanced RS. Experiments conducted on Epinions.com dataset showed that the accuracy of this combination is better than CF but worse than using trustenhanced alone. This result is because CF usually cannot find good neighbours to active users in sparse datasets, since it calculates similarity just according to some ratings. There are a lot of situations that two users have not rated items in a similar way but actually they are really similar. The reverse situation is also true; there are some situations that users have rated similar items in a similar way but actually they have not similar taste in other cases. In the other side, trust information is much reliable since they are expressed by users in a direct way and not calculated from ratings. Although rating coverage is improved by combining CF and trust-enhanced $\mathrm{RS}$, but this improvement is not such an excellent victory that let us carry the deterioration of accuracy in comparison with using trust-enhanced RS alone. So as a final conclusion, we can say that, hybrid recommender systems are not always preferable to single techniques and there are some situations as described in this paper that a single technique has better performance. Finally, it is worth noting that CF is not a good method to find similar users in sparse datasets when trust information is available.

\section{REFERENCES}

[1] A. Jøsang, S. Marsh and S. Pope, Exploring different types of trust propagation, LNCS 3986, (2006), 179-192.

[2] A.M. Rashid, G. Karypis and J. Riedl, Influence in ratings based recommender systems: an algorithmindependent approach, in: Proc. of SIAM International Conference on Data Mining, (2005).57-66.

[3] B.N Miller, J.A Konstan, and J. Riedl, PocketLens: toward a personal recommender system, ACM Trans. Inform. Syst. 22(3), (2002), 437-476.

[4] B.P Knijnenburg, M.C. Willemsen, Z. Gantner, H. Soncu and C. Newell, Explaining the user experience of recommender systems. User Model. User-Adap. Int. 22(4-5), (2012), 441-504.

[5] C. Hess, K. Stein and C. Schlieder, Trust-enhanced visibility for personalized document recommendations, in: Proc. of SAC2006, (2006), 1865-1869.

[6] G. Adomavicius and A. Tuzhilin, Toward the next generation of recommender systems: a survey of the state-of-the-art and possible extensions, IEEE Transactions on Knowledge and Data Engineering. 17(6), (2005), 734-749.

[7] H.J. Ahn, A new similarity measure for collaborative filtering to alleviate the new user cold-starting problem, Information Sciences 178, (2008) 37-51

[8] J. Golbeck, and J. Hendler, FilmTrust: movie recommendations using trust in web-based social networks, in: Proc. of CCNC2006, (2006), 282-286.

[9] J. Golbeck, Computing and applying trust in web-based social networks, $\mathrm{PhD}$ thesis (2005).

[10] J. Golbeck, Generating predictive movie recommendations from trust in social networks, LNCS 3986, (2006), 93-104.

[11] J. Herlocker, J. Konstan and J. Riedl, Explaining collaborative filtering recommendation, in: Proc. of CSCW2000, (2000), 241-250

[12] J. Herlocker, J. Konstan, L. Terveen and J. Riedl, Evaluating collaborative filtering recommender systems, ACM Trans. Inform. Syst. 22(1), (2004) 5-53.

[13] J.A. Konstan and J. Riedl, Recommender Systems: From Algorithms to User Experience. User Model. User-Adap. Int. 22(1-2), (2012), 101-123.

[14] L. Zhibing , A trust Enhanced Collaborative filtering recommender System, 5th International Conference on Computer Science and Education (ICCSE), (2010), 384 387.

[15] P. Bedi,H. Kaur and S. Marwaha, Trust based recommender system for the semantic web, in: Proc. of IJCAI-07, (2007) 2677-2682.

[16] P. Massa and A. Avesani, Trust-aware collaborative filtering for recommender systems, LNCS 3290, (2004), 492-508.

[17] P. Massa and B. Bhattacharjee, Using trust in recommender systems: an experimental analysis, LNCS 2995, (2004), 221-235. 
[18] P. Massa, A. Avesani and R. Tiella, A trust-enhanced recommender system application, Moleskiing, in: Proc. of SAC2005, (2005),1589-1593.

[19] P. Resnick and H.R. Varian, Recommender systems, Communications of the ACM, 40(3), (1997), 56-58.

[20] R. Burke, Hybrid recommender systems: survey and experiments, User Model. User-Adap. Int. 12(4), (2002), $31-370$

[21] R. Guha,, R. Kumar, P. Raghavan, and A. Tomkins, Propagation of trust and distrust, in: Proc. of WWW2004, (2004) 403-412.

[22] S. Ray and A. Mahanti, Improving Prediction Accuracy In Trust-Aware Recommender Systems, proceeding of the 43rd Hawaii international conference on systems sciences, 1-9 (2010).
[23] S.E. Middleton, H. Alani, N.R. Shadbolt and D.C.De Roure, Exploiting synergy between ontologies and recommender systems, Proc. of WWW2002 Semantic Web Workshop (2002).

[24] S.T. Park, D. Pennock, O. Madani, N. Good and D.De Coste, Naive filterbots for robust cold-start recommendations, Proc. of SIGKDD2006, (2006) , 699705.

[25] X. Su T.M. Khoshgoftaar, A Survey of Collaborative Filtering Techniques, Advances in Artificial Intelligence, vol. 2009, Article ID 421425, 19 pages, doi:10.1155/2009/421425.

[26] Z. Huang, H. Chen and D. Zeng, Applying associative retrieval techniques to alleviate the sparsity problem in collaborative filtering, ACM Trans. Inform. Syst. 22(1), (2004), 116-142 